\title{
Green Supply Chain Network Design under Multi-mode Production and Uncertainty
}

\author{
H. Heidari-Fathian ${ }^{1}$, S.H.R Pasandideh ${ }^{2, *}$
}

\begin{abstract}
A multi-periodic, multi-echelon green supply chain network consisting of manufacturing plants, potential distribution centers, and customers is developed. The manufacturing plants can provide the products in three modes including production in regular time, production in over time, or by subcontracting. The problem has three objectives including minimization of the total costs of the green supply chain network, maximization of the average safe inventory levels of the manufacturing plants and the distribution centers and minimization of the environmental impacts of the manufacturing plants in producing, holding and dispatching the products and also the environmental impacts of the distribution centers in holding and dispatching the products. The problem is first formulated as a mixed-integer mathematical model. Then, in order to solve the model, the augmented weighted Tchebycheff method is employed and its performance in producing the Pareto optimal solutions is compared with the goal attainment method.
\end{abstract}

Keywords: Green supply chain, Reliability, Multi-objective optimization, Augmented weighted Tchebycheff method.

Manuscript was received on 01/06/2017, revised on 09/09/2017 and accepted for publication on 16/09/2017.

\section{Introduction}

In a supply chain, several different activities are done until the customer demands are met. The manufacturing plants receive raw materials from suppliers, produce the products, and then send them to distribution centers, and the distribution centers are responsible for delivering products to the customers. In order to perform each of these activities, some special expends need to be made. Also, pollutions are made by most of these activities. Nowadays, economic and environmental problems are the main concerns of governments and have received considerable attention of many researchers for a number of years. An integrated review in the area of green supply chain was completed by Srivastava [1]. Sheu et al. [2] presented an optimization-based model to deal with integrated logistics operational problems of green-supply chain management. They considered factors such as the used-product return ratio and corresponding subsidies from governmental organizations for reverse logistics in the formulation of their model. Sundarakani et al. [3] examined the carbon footprint across supply chains and used analytical and finite difference methods to approximate their footprint model. Wang et al. [4] studied a supply chain network design problem with environmental concerns and proposed a multi-objective optimization model to capture the trade-off between the total cost and the environment influence. Mirzapour al-e-hashem el al. [5] developed a stochastic multi-objective stochastic programming approach to deal with a green

\footnotetext{
* Corresponding Author.

${ }^{1}$ Department of Industrial Engineering, Faculty of Engineering, Kharazmi University, Tehran, Iran, Email: std_fathian@khu.ac.ir.

2 Department of Industrial Engineering, Faculty of Engineering, Kharazmi University, Tehran, Iran, Email: shr_pasandideh@khu.ac.ir.
} 
supply chain planning under uncertainty. Chan et al. [6] demonstrated an effective incorporation of environmental issues into vendor-buyer coordination and developed a single-vendor multi-buyer coordination model that included both costs and environmental performance measures in its objective function. Sazvar et al. [7] proposed a new replenishment policy in a centralized supply chain for deteriorating items and considered environmental issues by considering several transportation vehicles producing various greenhouse gas levels. Fahimnia et al. [8] developed a unified optimization model for a closed-loop supply chain and evaluated the forward and reverse supply chain influences on the carbon footprint. Baud-Lavigne et al. [9] proposed some mathematical models for optimizing costs in a supply chain problem in which the environmental constraints were considered to control the carbon emissions. Azadeh et al. [10] used a multiobjective multi-period fuzzy linear programming model considering economic and environmental objectives for optimization of natural gas supply chain through a greenhouse gas reduction approach. Li et al. [11] examined a dual-channel green supply chain problem for analyzing the pricing and greening strategies for the chain members in both centralized and decentralized cases using the Stackelberg game model under a consistent pricing strategy. Kannan et al. [12] presented an integrated approach of fuzzy multi attribute utility theory and multi-objective programming for rating and selecting the best green suppliers considering economic and environmental criteria. Similar studies can also be found in Kadziński et al. [13], Niakan et al. [14], Kostin et al. [15], Xing et al. [16], Yu et al. [17] and Shaw et al. [18].

The weather pollution has a direct effect on the health of human beings and in many countries like Iran, the governments are forced to prorogate some days of the year in order to protect the health of their citizens, and, surely, these holidays have considerable costs for the governments. The environmental effects due to the activities of different echelons of a supply chain can be divided into three main categories: (i) The environmental effects due to production of products at manufacturing plants, (ii) the environmental effects due to handling and holding at warehouses of manufacturing plants and distribution centers and (iii) the environmental effects due to transportation of products among different echelons of a supply chain network. Beside these considerations, in competitive markets there are a lot of choices for customers in the selection of a company for meeting their needs. If a selected company cannot effectively provide and deliver the needs of the customers, the customer may decide to select another company for supplying its needs, resulting in loss of customer as well as credibility of the company. For this reason, all companies must be reliable to timely supply the needs of customers whenever they request.

The aim of our work here is to address the problem of pollution and reliability in supply chain networks by presenting a three-echelon green supply chain network consisting of manufacturing plants, distribution centers, and customers as demand points, where the number and location of the manufacturing plants are known, but there are a number of candidate locations for distribution centers that are subject to random failure and a subset of them must be selected based on fixed establishment costs and capacities in order to store the products and dispatch them to the customers. Also, the manufacturing plants can provide the products in three modes including regular time production, over time production or by subcontracting. The problem has three conflicting objectives. The first objective is to minimize the total supply chain costs including the production cost of the manufacturing plants, the fixed cost of establishing distribution centers, the setup cost of producing the products, the transportation cost of the products and the inventory holding cost of the products. The second objective is to maximize the average safe inventory levels of the manufacturing plants and the distribution centers and the last objective is to minimize the environmental impacts of the manufacturing plants and the distribution centers. The problem is first formulated as a three-objective mixed-integer programming model and then a multi-objective decision making (MODM) method, called augmented weighted Tchebycheff method, is employed 
to solve the problem. The applicability of the proposed solution method is evaluated by comparing it with the goal attainment method. Our contribution is to made in both the model and the solution approach. The available studies have not considered augmented weighted Tchebycheff method as a solving method and do not use a three-objective approach to the problem.

The remainder of our paper is organized as follows. The problem description and the mathematical model are given in Section 2. Section 3 discusses the solution method to solve the problem. The performance of the solution method is evaluated in Section 4. Finally, conclusions come in Section 5.

\section{The Problem}

\subsection{Problem Description, Assumptions and Notations}

The proposed supply chain includes manufacturing plants, distribution centers and demand points. Each manufacturing plant produces different types of products and dispatches them to the distribution centers or holds them in its warehouse considering some constraints related to the manufacturing plants including maximum available time to produce the products, the capacity of the manufacturing plants to transform the products to the distribution centers and also the total storage capacity of the plants. The manufacturing plant can provide the products in three ways with different costs including production in regular time, production in over time, and subcontracting. The distribution centers are the second echelon of the supply chain under investigation that receive the products from the manufacturing plants and store them in order to fulfill the demands of the customers that form final echelon of the supply chain. The assumptions related to the manufacturing plants and the distribution centers are a little different. The number and location of the manufacturing plants are known, while there are a number of candidate locations for the distribution centers and a subset of them must be selected based on the fixed establishment costs and their capacities in order to store the products and dispatch them to the customers. Another difference between the manufacturing plants and the distribution centers is that the reliability of the distribution centers in not certain, in other words, they do not operate perfectly all the time in term of dispatching the products to the demand point and they may fail to dispatch the products to the demand points in a period. The environmental impact of the manufacturing plants in producing, holding and dispatching the products and also the environmental impact of the distribution centers in holding and dispatching the products are taken into account in the proposed model. An escalating factor for different costs of the problem is also considered in the model.

With the points mentioned above as assumptions, the problem is formulated as a three-objective mixed integer model to design a green supply chain network such that the first objective is to minimize the total supply chain costs including the production cost of the manufacturing plants, the fixed cost of establishing warehouses, the setup cost of producing the products, the transportation cost of the products and the inventory holding cost of the products. The second objective is to maximize the average safe inventory levels of the manufacturing plants and the distribution centers. Eventually, the last objective function minimizes the environmental impacts of the manufacturing plants and the distribution centers.

\section{- Indices}

$M:$ Number of manufacturing plants indexed by $m$

$\mathrm{J}$ : Number of potential locations of distribution centers indexed by $j$ 
$I:$ Number of customers indexed by $i$

$K$ : Number of finished products indexed by $k$

$T:$ Number of time periods indexed by $t$ with length of $\tau$.

\section{- Parameters}

$c_{m t}^{k}$ : Unit production cost of product $k$ produced by manufacturing plant $m$ in period $t$

$d_{j m t}^{k}$ : Unit transportation cost of product $k$ from manufacturing plant $m$ to distribution center $j$ in

$d_{i j t}^{k}$ : Unit transportation cost of product $k$ from distribution center $j$ to customer $i$ in period $t$

$C A_{m t}^{k}$ : $\quad$ Cost of producing product $k$ in manufacturing plant $m$ in period $t$

$H D_{j t}^{k}$ : Unit inventory holding cost of product $k$ in distribution center $j$ in period $t$

$H P_{m t}^{k}$ : Unit inventory holding cost of product $k$ in manufacturing plant $m$ in period $t$

$C S_{i t}^{k}$ : Unit shortage cost of product $k$ in supplying the demand of customer $i$ in period $t$

$f_{j}: \quad$ Fixed cost of establishing distribution center $j$

$P T_{m}^{k}$ : $\quad$ Production time needed in manufacturing plant $m$ to produce one unit of product $k$ per period

$A_{m}^{k}: \quad$ Time of producing product $k$ in manufacturing plant $m$ per period

$T T_{m t}$ : Total available production time for manufacturing plant $m$ to produce product $k$ in period $t$

$W_{j}$ : Total storage capacity available in distribution center $j$ to store products in a period

$W_{m}$ : Storage capacity available for manufacturing plant $m$ to store products in a period

$R_{m}^{k}$ : Total transportation capacity available for manufacturing plant $m$ to dispatch product $k$ in a period

$D C_{i t}^{k}$ : Demand of product $k$ by customer $i$ in period $t$

$V_{k}$ : $\quad$ Volume of one unit of product $k$

$S I_{j}^{k}: \quad$ Safe inventory level of product $k$ in distribution center $j$ in a period

$S I_{m}^{k}$ : Safe inventory level of product $k$ in manufacturing plant $m$ in a period

$A T S_{m t}$ : Available Subcontracting time at manufacturing plant $m$ in period $t$

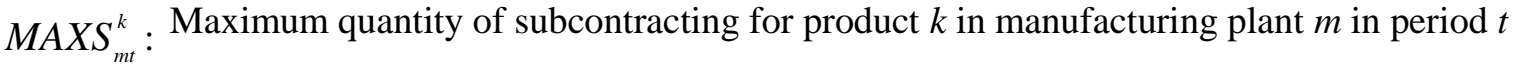

$P T S_{m}^{k}$ : Subcontracting time needed in manufacturing plant $m$ for providing one unit of product $k$ per period

$E F_{x}$ : $\quad$ Escalating factor for transportation cost of products from distribution centers to customers

$E F_{u}: \quad$ Escalating factor for transportation cost of products from manufacturing plants to distribution centers

$E F_{h d}: \quad$ Escalating factor for inventory holding cost in distribution centers

$E F_{z}: \quad$ Escalating factor for cost of producing products

$E F_{q r}$ : Escalating factor for regular production cost 
$E F_{q o}: \quad$ Escalating factor for over time production cost

$E F_{q s}: \quad$ Escalating factor for subcontracting production cost

$E F_{h p}: \quad$ Escalating factor for inventory holding cost in manufacturing plants

$E F_{s h}: \quad$ Escalating factor for shortage cost of products

$e p_{m}^{k}$ : $\quad$ Rate of carbon emission to produce one unit of product $k$ in manufacturing plant $m$ in a period

$e s_{m}^{k}$ : $\quad$ Rate of carbon emission for subcontracting one unit of product $k$ in manufacturing plant $m$ in a period

$e h_{j}^{k}$ : $\quad$ Rate of carbon emission to hold one unit of product $k$ in distribution center $j$ in a period

$e m_{m}^{k}$ : Rate of carbon emission to hold one unit of product $k$ in manufacturing plant $m$ in a period

$e m t d_{j m}^{k}$ : Rate of carbon emission to dispatch one unit of product $k$ from manufacturing plant $m$ to distribution center $j$ in a period

$\operatorname{edtc}_{i j}^{k}$ : Rate of carbon emission to dispatch one unit of product $k$ from distribution center $j$ to customer $i$ in a period.

\section{- Decision variables}

$Z_{m t}^{k}$ : A binary variable equal to 1 if product $k$ is produced by manufacturing plant $m$ in period $t$;

$Y_{j}: \quad$ A binary variable equal to 1 if distribution center $j$ is established; 0 , otherwise

$Q R_{m t}^{k}$ : Quantity of product $k$ produced by manufacturing plant $m$ in over time of period $t$

$Q O_{m t}^{k}$ : Quantity of product $k$ produced by manufacturing plant $m$ in regular time of period $t$

$Q S_{m t}^{k}$ : Subcontracting quantity for product $k$ produced at manufacturing plant $m$ in period $t$

$U_{j m t}^{k}$ : Quantity of product $k$ transported from manufacturing plant $m$ to distribution center $j$ in period $t$

$X_{i j t}^{k}$ : Quantity of product $k$ transported from distribution center $j$ to customer $i$ in period $t$

$S_{i t}^{k}: \quad$ Shortage quantity of product $k$ for demand of customer $i$ in period $t$

$I_{m t}^{k}$ : Inventory level of product $k$ in manufacturing plant $m$ at the end of period $t$

$I_{j t}^{k}: \quad$ Inventory level of product $k$ in distribution center $m$ at the end of period $t$

$S H_{m t}^{k}$ : Short safe inventory level of product $k$ in manufacturing plant $m$ at the end of period $t$

$S H_{j t}^{k}$ : Short safe inventory level of product $k$ in distribution center $j$ at the end of period $t$.

\subsection{Mathematical Model}

Here, a mathematical model is proposed for the problem. We do this in two stages. In the first stage, the objectives are formulated and then the constraints are explained. 


\subsubsection{Objective Functions}

Minimization of the total costs of the proposed green supply chain network is the first objective. The objective function is formulated as equation (1) below. According to this equation, the components of the cost objective function include the fixed cost of establishing the distribution centers, the transportation cost of the products from the manufacturing plants to the distribution centers, the setup cost of producing the products, the cost of holding the products in the distribution centers, transportation cost of products from the distribution centers to the demand points, production cost of the manufacturing plants in regular time, production cost of the manufacturing plants in over time, subcontracting cost of the products, the end inventory holding cost of the manufacturing plants, and the shortage cost of customers' demands, respectively from left to right:

$$
\begin{aligned}
\operatorname{Min} Z_{1}= & \sum_{j=1}^{J} f_{j} Y_{j}+\sum_{t=1}^{T} \sum_{m=1}^{M} \sum_{k=1}^{K} \sum_{j=1}^{J} d_{j m t}^{k} U_{j m t}^{k}\left(1+E F_{u}\right)^{t}+\sum_{m=1}^{M} \sum_{t=1}^{T} \sum_{k=1}^{K} C A_{m t}^{k} Z_{m t}^{k}\left(1+E F_{z}\right)^{t}+\sum_{j=1}^{J} \sum_{t=1}^{T} \sum_{k=1}^{K} H D_{j t}^{k} I_{j t}^{k}\left(1+E F_{h d}\right)^{t} \\
& +\sum_{t=1}^{T} \sum_{i=1}^{T} \sum_{k=1}^{K} \sum_{j=1}^{J} d_{i j t}^{k} X_{i j t}^{k}\left(1+E F_{x}\right)^{t}+\sum_{m=1}^{M} \sum_{t=1}^{T} \sum_{k=1}^{K} c r_{m t}^{k} Q R_{m t}^{k}\left(1+E F_{q r}\right)^{t}+\sum_{m=1}^{M} \sum_{t=1}^{T} \sum_{k=1}^{K} c o_{m t}^{k} Q O_{m t}^{k}\left(1+E F_{q o}\right)^{t} \\
& +\sum_{m=1}^{M} \sum_{t=1}^{T} \sum_{k=1}^{K} c s_{m t}^{k} Q S_{m t}^{k}\left(1+E F_{q s}\right)^{t}+\sum_{m=1}^{M} \sum_{t=1}^{T} \sum_{k=1}^{K} H P_{m t}^{k} I_{m t}^{k}\left(1+E F_{h p}\right)^{t}+\sum_{i=1}^{T} \sum_{t=1}^{T} \sum_{k=1}^{K} c s h_{i t}^{k} S_{i t}^{k}\left(1+E F_{s}\right)^{t}
\end{aligned}
$$

The second objective is to maximize the average safe inventory levels of the manufacturing plants and the distribution centers. The average safe inventory level of the manufacturing plant $m$ in period $t$ is calculated by detracting the ratio of short safe inventory level of product $k$ in manufacturing plant $m$, at period $t$, over the quantity of the safe inventory of product $k$ in manufacturing plants $m$ starting from 1. Similar considerations can also be made for the distribution centers. The second objective function is formulated in Equations (2).

$\operatorname{Max} Z_{2}=\sum_{m=1}^{M} \sum_{t=1}^{T} \sum_{k=1}^{K}\left(1-\frac{S H_{m t}^{k}}{S I_{m}^{k}}\right)+\sum_{j=1}^{J} \sum_{t=1}^{T} \sum_{k=1}^{K} Y_{j}\left(1-\frac{S H_{j t}^{k}}{S I_{j}^{k}}\right)$.

The third objective is to minimize the environmental impacts of the manufacturing plants and the distribution centers. As shown in equation (3) below, the first component of the objective relates to the total environmental impacts of the manufacturing plants due to producing the products in regular and over time and the second term calculate the total environmental impacts of subcontracting the products, the third and fourth terms of the objective function represent the environmental impacts due to holding the products in distribution centers and manufacturing plants, respectively. Finally, the fifth and sixth terms relate to the environmental impacts due to the transportation of the products from manufacturing plants to distribution centers and also from distribution centers to demand points, respectively:

$$
\begin{aligned}
\operatorname{Min}_{3}= & \sum_{m=1}^{M} \sum_{t=1}^{T} \sum_{k=1}^{K} e p_{m}^{k}\left(Q R_{m t}^{k}+Q O_{m t}^{k}\right)_{+} \sum_{m=1}^{M} \sum_{t=1}^{T} \sum_{k=1}^{K} e s_{m}^{k} Q S_{m t}^{k}+\sum_{t=1}^{T} \sum_{m=1}^{M} \sum_{k=1}^{K} \sum_{j=1}^{J} e m t d_{j m}^{k} U_{j m t}^{k} \\
& +\sum_{m=1}^{M} \sum_{t=1}^{T} \sum_{k=1}^{K} e m_{m}^{k} I_{m t}^{k}+\sum_{j=1}^{J} \sum_{t=1}^{T} \sum_{k=1}^{K} e h_{j}^{k} I_{j t}^{k}+\sum_{t=1}^{T} \sum_{i=1}^{I} \sum_{k=1}^{K} \sum_{j=1}^{J} e d t c_{i j}^{k} X_{i j t}^{k} .
\end{aligned}
$$




\subsubsection{Formulation of Constraints}

Here, we formulate and describe the constraints.

\section{- Constraints on available time}

There is a specific time for producing the products in every period and the total required time to produce the products including the production time and also setup time in the manufacturing plants cannot exceed the total available time:

$$
\sum_{k=1}^{K} P T_{m}^{k} \cdot\left(Q R_{m t}^{k}+Q O_{m t}^{k}\right)+\sum_{k=1}^{K} A_{m}^{k} \cdot Z_{m t}^{k} \leq T T_{m t}, \quad \forall m, t .
$$

\section{- Constraints on demand}

Relating to demands of the customers, the quantity of a product dispatched to each customer in a period cannot be more than its demand:

$$
\sum_{j=1}^{J} X_{i j t}^{k} \leq D C_{i t}^{k}, \quad \forall i, t, k
$$

\section{- Constraints on capacity}

Constraints in equations (6) and (7) below relate to the storage capacity of the potential distribution centers that restrict the amount of the products transformed from manufacturing plants to distribution centers and also the inventory of the distribution centers at the end of every period, respectively:

$$
\begin{array}{ll}
\sum_{k=1}^{K} \sum_{m=1}^{M} V_{k} \cdot U_{j m t}^{k} \leq W_{j} Y_{j}, & \forall j, t, \\
\sum_{k=1}^{K} V_{k} \cdot I_{j t}^{k} \leq W_{j} Y_{j}, & \forall j, t .
\end{array}
$$

Constraints (8) and (9) below are capacity constraints related to the manufacturing plants. Constraints in equation (8) express that the manufacturing plants have a limited capacity for dispatching the products to the distribution centers and constraints (9) restrict the inventory of the manufacturing plants at the end of every period:

$$
\begin{array}{ll}
\sum_{j=1}^{J} U_{j m t}^{k} \leq R_{m}^{k} Z_{m t}^{k}, & \forall k, t, m, \\
\sum_{k=1}^{K} V_{k} \cdot I_{m t}^{k} \leq W_{m}, & \forall m, t .
\end{array}
$$




\section{- Constraints on reliability}

As mentioned before, the distribution centers do not operate perfectly all the time in term of dispatching products to the demand point and they may fail to dispatch products to the demand point in a period. This failing follows an exponential distribution with a mean of $\lambda_{j t}$.

Accordingly, the reliability of the distribution centers to dispatch the products to the customers in a period is $e^{-\lambda_{j t} \tau}$ and the average number of product $k$ dispatched from potential distribution center $j$ to customer $i$ is $\left(e^{-\lambda_{j t} \tau}, X_{i j t}^{k}\right)$. Equation (10) specify the lower and upper bounds of the average total number of products dispatched to customers.

$$
L B \leq \sum_{t=1}^{T} \sum_{i=1}^{I} \sum_{k=1}^{K} \sum_{j=1}^{J} \mathrm{e}^{-\lambda_{j i} \tau} \cdot X_{i j t}^{k} \leq U B
$$

\section{- Constraints on inventory}

$$
\begin{array}{ll}
I_{m t}^{k}=I_{m t-1}^{k}+Q R_{m t}^{k}+Q O_{m t}^{k}+Q S_{m t}^{k}-\sum_{j=1}^{J} U_{j m t}^{k}, & \forall m, k, t \\
I_{j t}^{k}=I_{j t-1}^{k}+\sum_{m=1}^{M} U_{j m t}^{k}-\sum_{i=1}^{I} X_{i j t}^{k}, & \forall j, k, t
\end{array}
$$

The constraints in equations (11) and (12) are balance equations for inventory of the products at the manufacturing plants and distribution centers, respectively.

\section{- Constraints on contractor}

$$
\begin{array}{ll}
\sum_{k=1}^{K} P T S_{m}^{k} \cdot Q S_{m t}^{k} \leq A T S_{m t}, & \forall m, t \\
Q S_{m t}^{k} \leq M A X S_{m t}^{k}, & \forall k, m, t
\end{array}
$$

Equations (13) and (14) are related to the contractor and respectively specify the available Subcontracting time and maximum amount of subcontracting for products in each manufacturing plant.

\section{- Constraints on shortage}

Next balance equation is related to the shortages of the demands of the customers that is shown in equation (15).

$$
S_{i t}^{k}=S_{i t-1}^{k}+D C_{i t}^{k}-\sum_{j=1}^{J} X_{i j t}^{k}, \quad \forall i, k, t
$$




\section{- Constraints on short safe inventory}

Constraints (16) and (17) below calculate the amounts of short safe inventory of products at the manufacturing plants and the distribution centers, respectively. As shown, if the quantity of the safe inventory is greater than the inventory level, the amount of the short safe inventory is equal to their difference; otherwise, the amounts is equal to zero:

$$
\begin{aligned}
& S H_{m t}^{k}=\left\{\begin{array}{ll}
S I_{m}^{k}-I_{m t}^{k}, & S I_{m}^{k} \geq I_{m t}^{k}, \\
0, & \text { otherwise, }
\end{array} \quad \forall m, k, t\right. \\
& S H_{j t}^{k}=\left\{\begin{array}{ll}
S I_{j}^{k}-I_{j t}^{k}, & S I_{j}^{k} \geq I_{j t}^{k}, \\
0, & \text { otherwise. }
\end{array} \quad \forall j, k, t\right.
\end{aligned}
$$

\section{- Binary and non-negativity constraints on decision variables}

$$
\begin{array}{ll}
Y_{j}, Z_{m t}^{k} \in\{0,1\}, & \forall m, j, k, t \\
\left(\begin{array}{l}
Q R_{m t}^{k}, Q O_{m t}^{k}, Q S_{m t}^{k}, I_{m t}^{k}, S_{i t}^{k} \\
X_{i j t}^{k}, U_{j m t}^{k}, I_{j t}^{k}, \mathrm{SH}_{\mathrm{mt}}^{\mathrm{k}}, \mathrm{SH}_{\mathrm{jt}}^{\mathrm{k}}
\end{array}\right) \geq 0, & \forall m, j, i, k, t .
\end{array}
$$

Thus, the complete model for solving the green supply chain problem is formulated as follows:

$$
\begin{aligned}
\operatorname{Min}_{1}= & \sum_{j=1}^{J} f_{j} Y_{j}+\sum_{t=1}^{T} \sum_{m=1}^{M} \sum_{k=1}^{K} \sum_{j=1}^{J} d_{j m t}^{k} U_{j m t}^{k}\left(1+E F_{u}\right)^{t}+\sum_{m=1}^{M} \sum_{t=1}^{T} \sum_{k=1}^{K} C A_{m t}^{k} Z_{m t}^{k}\left(1+E F_{z}\right)^{t}+\sum_{j=1}^{J} \sum_{t=1}^{T} \sum_{k=1}^{K} H D_{j t}^{k} I_{j t}^{k}\left(1+E F_{h d}\right)^{t} \\
& +\sum_{t=1}^{T} \sum_{i=1}^{I} \sum_{k=1}^{K} \sum_{j=1}^{J} d_{j i t}^{k} X_{i j t}^{k}\left(1+E F_{x}\right)^{t}+\sum_{m=1}^{M} \sum_{t=1}^{T} \sum_{k=1}^{K} c r_{m t}^{k} Q R_{m t}^{k}\left(1+E F_{q r}\right)^{t}+\sum_{m=1}^{M} \sum_{t=1}^{T} \sum_{k=1}^{K} c o_{m t}^{k} Q O_{m t}^{k}\left(1+E F_{q o}\right)^{t} \\
& +\sum_{m=1}^{M} \sum_{t=1}^{T} \sum_{k=1}^{K} c s_{m t}^{k} Q S_{m t}^{k}\left(1+E F_{q s}\right)^{t}+\sum_{m=1}^{M} \sum_{t=1}^{T} \sum_{k=1}^{K} H P_{m t}^{k} I_{m t}^{k}\left(1+E F_{h p}\right)^{t}+\sum_{i=1}^{I} \sum_{t=1}^{T} \sum_{k=1}^{K} c s h_{i t}^{k} S_{i t}^{k}\left(1+E F_{s}\right)^{t} \\
\operatorname{Max}_{2}= & \sum_{m=1}^{M} \sum_{t=1}^{T} \sum_{k=1}^{K}\left(1-\frac{S H_{m t}^{k}}{S I_{m}^{k}}\right)+\sum_{j=1}^{J} \sum_{t=1}^{T} \sum_{k=1}^{K} Y_{j}\left(1-\frac{S H_{j t}^{k}}{S I_{j}^{k}}\right) \\
\operatorname{MinZ}_{3}= & \sum_{m=1}^{M} \sum_{t=1}^{T} \sum_{k=1}^{K} e p_{m}^{k}\left(Q R_{m t}^{k}+Q O_{m t}^{k}\right)_{+} \sum_{m=1}^{M} \sum_{t=1}^{T} \sum_{k=1}^{K} e S_{m}^{k}\left(Q S_{m t}^{k}\right)+\sum_{t=1}^{T} \sum_{m=1}^{M} \sum_{k=1}^{K} \sum_{j=1}^{J} e m t d_{j m}^{k} U_{j m t}^{k} \\
& +\sum_{m=1}^{M} \sum_{t=1}^{T} \sum_{k=1}^{K} e m_{m}^{k} I_{m t}^{k}+\sum_{j=1}^{J} \sum_{t=1}^{T} \sum_{k=1}^{K} e h_{j}^{k} I_{j t}^{k}+\sum_{t=1}^{T} \sum_{i=1}^{I} \sum_{k=1}^{K} \sum_{j=1}^{J} e d t c_{i j}^{k} X_{i j t}^{k}
\end{aligned}
$$


s.t.

$\sum_{k=1}^{K} P T_{m}^{k} \cdot\left(Q R_{m t}^{k}+Q O_{m t}^{k}\right)+\sum_{k=1}^{K} A_{m}^{k} \cdot Z_{m t}^{k} \leq T T_{m t}, \quad \forall m, t$

$\sum_{j=1}^{J} X_{i j t}^{k} \leq D C_{i t}^{k}, \quad \forall i, t, k$

$\sum_{k=1}^{K} \sum_{m=1}^{M} V_{k} \cdot U_{j m t}^{k} \leq W_{j} Y_{j}, \quad \forall j, t$

$\sum_{k=1}^{K} V_{k} \cdot I_{j t}^{k} \leq W_{j} Y_{j}, \quad \forall j, t$

$\sum_{j=1}^{J} U_{j m t}^{k} \leq R_{m}^{k} Z_{m t}^{k}, \quad \forall k, t, m$

$\sum_{k=1}^{K} V_{k} \cdot I_{m t}^{k} \leq W_{m}, \quad \forall m, t$

$L B \leq \sum_{t=1}^{T} \sum_{i=1}^{I} \sum_{k=1}^{K} \sum_{j=1}^{J} \mathrm{e}^{-\lambda_{j} \tau} \cdot X_{i j t}^{k} \leq U B$

$I_{m t}^{k}=I_{m t-1}^{k}+Q R_{m t}^{k}+Q O_{m t}^{k}+Q S_{m t}^{k}-\sum_{j=1}^{J} U_{j m t}^{k}, \quad \forall m, k, t$

$I_{j t}^{k}=I_{j t-1}^{k}+\sum_{m=1}^{M} U_{j m t}^{k}-\sum_{i=1}^{I} X_{i j t}^{k}, \quad \forall j, k, t$

$\sum_{k=1}^{K} \mathrm{PTS}_{m}^{k} \cdot \mathrm{QS}_{m t}^{k} \leq A T S_{m t}, \quad \forall m, t$

$\mathrm{QS}_{m t}^{k} \leq M A X S_{m t}^{k}, \quad \forall k, m, t$

$S_{i t}^{k}=S_{i t-1}^{k}+D C_{i t}^{k}-\sum_{j=1}^{J} X_{i j t}^{k}, \quad \forall i, k, t$

$S H_{m t}^{k}=\left\{\begin{array}{ll}S I_{m}^{k}-I_{m t}^{k}, & S I_{m}^{k} \geq I_{m t}^{k}, \\ 0, & \text { otherwise, }\end{array} \forall m, k, t\right.$

$S H_{j t}^{k}=\left\{\begin{array}{ll}S I_{j}^{k}-I_{j t}^{k}, & S I_{j}^{k} \geq I_{j t}^{k}, \\ 0, & \text { otherwise, }\end{array} \forall j, k, t\right.$

$Y_{j}, Z_{m t}^{k} \in\{0,1\}, \quad \forall m, j, k, t$

$\left(\begin{array}{l}Q R_{m t}^{k}, Q O_{m t}^{k}, Q S_{m t}^{k}, I_{m t}^{k}, S_{i t}^{k} \\ X_{i j t}^{k}, U_{j m t}^{k}, I_{j t}^{k}, S H_{m t}^{k}, S H_{j t}^{k}\end{array}\right) \geq 0, \quad \forall m, j, i, k, t$. 


\section{Solution Method}

There are a variety of methods such as $\varepsilon$-constraint [19], LP-metric [20], goal programming [21], and evolutionary algorithms [22] to solve multi-objective problems. In multi-objective mathematical programming, there is a number of conflicting objectives that should be optimized simultaneously and there is no single optimal solution that simultaneously optimizes all the objective functions. Indeed, in these types of problems one objective cannot be improved without deteriorating the performance of at least one of the others. Such solutions are called Pareto-optimal solutions. Here, we employ the augmented weighted Tchebycheff method to obtain Pareto-optimal solutions. This method is a scalarization approach for solving multi-objective optimization problems that uses an exact solution procedure for producing Pareto-optimal solutions [23], [24]. The objective functions are combined into a single one, each having a normalized portion in the cumulative objective function on accordance with the associated weight. The general mathematical form of the augmented weighted Tchebycheff (AWT) method is as follows:

$\operatorname{Min}\left\{\gamma+\rho \sum_{g=1}^{G}\left(\frac{f_{g}^{*}-f_{g}}{f_{g}^{*}}\right)\right\}$

s.t.

$w_{g} \cdot\left(\frac{f_{g}^{*}-f_{g}}{f_{g}^{*}}\right) \leq \gamma, \quad g=1,2, \ldots, G$

$x \in S$,

where $\rho$ is a small positive number, $S$ is the feasible space of the original problem, $f_{g}$ is the objective function $g$ and $f_{g}^{*}$ is its optimal solution, $w_{g}$ is the weight factor of the $g$ th objective function such that $\sum_{i=1}^{g}\left|w_{i}\right|=1$ and $\gamma$ is an unrestricted variable. The weight factors in the AWT method will be determined by Decision Makers (DMs), such as manager or experts, and it is increased by DM when the importance of the objective function is greater. It should be noted that one can employ systematic approaches, for example AHP and ANP, for selecting the weights.

Also, we employ Goal Attainment (GA) method [23] for comparison with the weighted Tchebycheff method. The method requires that the DM gives a goal vector $b$ and a vector of weight $W$. This approach, has a wide application in converting multi-objective optimization problems into single-objective optimization problems. The mathematical formulation of the problem is:

$\operatorname{Min} z$

s.t.

$g_{j}(x) \leq 0, \quad \forall j$

$f_{i}(x)-w_{i} z \geq b_{i}, \quad i=1,2, \ldots, k$

$W>0$,

where $z$ is a scalar variable unrestricted in sign, $W$ is generally normalized so that $\sum_{i=1}^{k}\left|w_{i}\right|=1, k$ is the number of objective functions, and $f_{i}$ is the objective function $i$. 


\section{Performance Evaluation}

Our aim is to evaluate the performance of the augmented weighted Tchebycheff method. For a suitable evaluation, this method must be compared with a conventional MODM method that here the Goal Attainment (GA) method is selected for this purpose. The comparison is performed based on four criteria including the value of the objective functions, and the required computing times for solving the model. It is important to say that the GA method is considered as a powerful tool among MODM methods [23]. The main reason for popularity of the GA method is its ability to reach the goals or even better than the goals.

Thirty different numerical examples are randomly generated considering different sizes for manufacturing plants, distribution centers, customers and time periods. The generated problems are shown in Table1. The examples are solved on a laptop with core (TM) i5, $2.40 \mathrm{GHz}$, RAM $4 \mathrm{Mb}$ using GAMS software. The input parameters of the proposed mathematical model are randomly generated using uniform distributions presented in Table 2. Table 3 shows the results of this performance evaluation. It can be seen that, in term of the first objective function, except for problems 5, 11, 19 and 22 on which the results of GA method are better than AWT method, the Pareto optimal solutions obtained by AWT method are better than GA. Regarding the second criterion (i.e., the value of the second objective function), in all problems except for problems 15 , AWT method works better. Also, except for problems 5, 9, 17, 24 and 28, the produced Pareto optimal solutions obtained by AWT method are better than GA method in term of the second objective function. Finally, the last two column of Table 3 show that the computing time of AWT method is less than GA method. Based on these results, it is clear that the performance of the AWT method is better than the GA method.

\section{Conclusion}

We developed a mathematical model for a green supply chain network consisting of manufacturing plants, potential distribution centers and customers. The problem was first formulated into a mixed-integer mathematical model with three potentially conflicting objectives including minimization of the total costs of the green supply chain network, maximization of the average safe inventory levels of the manufacturing plants and distribution centers and minimization of the environmental impacts of the manufacturing plants in producing, holding and dispatching the products and also the environmental impacts of the distribution centers in holding and dispatching the products. In order to solve the model, the augmented weighted Tchebycheff method was employed as a solution method and its performance in producing the Pareto optimal solutions was compared with the ones obtained by the goal attainment method using thirty different numerical examples considering four criteria including the values of the three objective functions and also the required computating times. The results showed outperformance of the augmented weighted Tchebycheff method. 
Table 1. Generated problems

\begin{tabular}{|c|c|c|c|c|c|c|}
\hline $\begin{array}{c}\text { Problem } \\
\text { code }\end{array}$ & Problem number & $\begin{array}{l}\text { No. of } \\
\text { MPs }\end{array}$ & $\begin{array}{c}\text { No. of } \\
\text { DCs }\end{array}$ & $\begin{array}{c}\text { No. of } \\
\text { Customers }\end{array}$ & $\begin{array}{c}\text { No. of } \\
\text { products }\end{array}$ & $\begin{array}{l}\text { No. of } \\
\text { periods }\end{array}$ \\
\hline 1 & $7-11-3-6-7$ & 7 & 11 & 3 & 6 & 7 \\
\hline 2 & $4-12-6-4-8$ & 4 & 12 & 6 & 4 & 8 \\
\hline 3 & $5-5-3-3-15$ & 5 & 5 & 3 & 3 & 15 \\
\hline 4 & $4-16-5-2-10$ & 4 & 16 & 5 & 2 & 10 \\
\hline 5 & $5-12-4-4-8$ & 5 & 12 & 4 & 4 & 8 \\
\hline 6 & $6-24-4-6-13$ & 6 & 24 & 4 & 6 & 13 \\
\hline 7 & $5-10-9-4-11$ & 5 & 10 & 9 & 4 & 11 \\
\hline 8 & $13-19-6-3-13$ & 13 & 19 & 6 & 3 & 13 \\
\hline 9 & $3-8-7-5-20$ & 3 & 8 & 7 & 5 & 20 \\
\hline 10 & $3-7-5-2-11$ & 3 & 7 & 5 & 2 & 11 \\
\hline 11 & $4-10-3-3-9$ & 4 & 10 & 3 & 3 & 9 \\
\hline 12 & $6-6-2-4-14$ & 6 & 6 & 2 & 4 & 14 \\
\hline 13 & $7-14-5-4-6$ & 7 & 14 & 5 & 4 & 6 \\
\hline 14 & $3-8-5-2-18$ & 3 & 8 & 5 & 2 & 18 \\
\hline 15 & $4-16-2-5-3$ & 4 & 16 & 2 & 5 & 3 \\
\hline 16 & $9-22-8-5-7$ & 9 & 22 & 8 & 5 & 7 \\
\hline 17 & $6-6-3-5-19$ & 6 & 6 & 3 & 5 & 19 \\
\hline 18 & 8-11-11-4-13 & 8 & 11 & 11 & 4 & 13 \\
\hline 19 & 2-14-6-8-4 & 2 & 14 & 6 & 8 & 4 \\
\hline 20 & $8-12-7-4-9$ & 8 & 12 & 7 & 4 & 9 \\
\hline 21 & $8-17-7-9-4$ & 8 & 17 & 7 & 9 & 4 \\
\hline 22 & $4-9-4-5-9$ & 4 & 9 & 4 & 5 & 9 \\
\hline 23 & $3-11-6-6-5$ & 3 & 11 & 6 & 6 & 5 \\
\hline 24 & $5-15-4-5-4$ & 5 & 15 & 4 & 5 & 4 \\
\hline 25 & $7-7-5-2-14$ & 7 & 7 & 5 & 2 & 14 \\
\hline 26 & $3-3-4-5-12$ & 3 & 3 & 4 & 5 & 12 \\
\hline 27 & $5-12-7-3-10$ & 5 & 12 & 7 & 3 & 10 \\
\hline 28 & $6-12-6-3-7$ & 6 & 12 & 6 & 3 & 7 \\
\hline 29 & $3-8-13-4-14$ & 3 & 8 & 13 & 4 & 14 \\
\hline 30 & $4-14-7-5-9$ & 4 & 14 & 7 & 5 & 9 \\
\hline
\end{tabular}


Table 2. Values of the parameters

\begin{tabular}{cc|cc|cc}
\hline parameter & value & parameter & Value & parameter & value \\
\hline$d_{j m t}^{k}$ & $(10,24)$ & $S I_{m}^{k}$ & $(100,200)$ & $P T_{m}^{k}$ & $(0.1,0.9)$ \\
$d_{i j t}^{k}$ & $(3,10)$ & $\mathrm{SI}_{j}^{k}$ & $(10,50)$ & $T T_{m t}$ & $(70,320)$ \\
$C A_{m t}^{k}$ & $(100,350)$ & $e m_{m}^{k}$ & $(2,5)$ & $f_{j}$ & $(4000,10000)$ \\
$M A X S^{\prime}$ & $(10,15)$ & $e d t c_{i j}^{k}$ & $(5,8)$ & $R_{m}^{k}$ & $(45,80)$ \\
$A T S_{m t}$ & $(30,60)$ & $e p_{m}^{k}$ & $(6,10)$ & $D C_{i t}^{k}$ & $(15,60)$ \\
$c r_{m t}^{k}$ & $(1,6)$ & $e h_{j}^{k}$ & $(2,5)$ & $V_{k}$ & $(0.8,1.5)$ \\
$c O_{m t}^{k}$ & $(2,12)$ & $e m t d_{j m}^{k}$ & $(5,8)$ & $W_{j}$ & $(50,400)$ \\
$c s_{m t}^{k}$ & $(2,14)$ & $e S_{m}^{k}$ & $(2,5)$ & $W_{m}$ & $(500,1200)$ \\
$c s h_{i t}^{k}$ & $(80,180)$ & $P T P_{m}^{k}$ & $(0.15,1.1)$ & $\lambda_{j t}$ & $(0.02,0.05)$ \\
$A_{m}^{k}$ & $(10,35)$ & $H D_{j t}^{k}$ & $(2,5)$ & $H P_{m t}^{k}$ & $(4,9)$ \\
\hline
\end{tabular}


Table 3. Results of the performance evaluation

\begin{tabular}{|c|c|c|c|c|c|c|c|c|c|}
\hline \multirow{2}{*}{$\begin{array}{l}\text { problem } \\
\text { number }\end{array}$} & \multirow{2}{*}{ Problem code } & \multicolumn{2}{|c|}{$\mathrm{Z}_{1}$} & \multicolumn{2}{|c|}{$\mathrm{Z}_{2}$} & \multicolumn{2}{|c|}{$\mathrm{Z}_{3}$} & \multicolumn{2}{|c|}{ CPU time } \\
\hline & & AWT & GA & AWT & GA & AWT & GA & AWT & GA \\
\hline 1 & $7-11-3-6-7$ & $7,906,817.09$ & $8,073,579.05$ & 130.41 & 124.05 & $118,645.76$ & $120,653.62$ & 76.61 & 132.4 \\
\hline 2 & $4-12-6-4-8$ & $24,874,538.33$ & $25,399,164.96$ & 155.34 & 147.76 & $84,944.10$ & $86,381.61$ & 47.50 & 83.56 \\
\hline 3 & $5-5-3-3-15$ & $22,524,104.17$ & $22,999,158.00$ & 241.92 & 230.12 & $151,753.33$ & $154,321.47$ & 21.54 & 48.33 \\
\hline 4 & $4-16-5-2-10$ & $27,861,680.00$ & $28,449,308.16$ & 87.95 & 83.66 & $57,818.92$ & $58,797.39$ & 51.28 & 132.9 \\
\hline 5 & $5-12-4-4-8$ & $19,952,377.50$ & $19,943,191.28$ & 201.48 & 191.66 & $60,699.69$ & $60,516.78$ & 168.33 & 244.8 \\
\hline 6 & $6-24-4-6-13$ & $6,437,710.83$ & $6,573,488.00$ & 185.20 & 176.17 & $71,972.54$ & $73,190.54$ & 46.67 & 88.12 \\
\hline 7 & $5-10-9-4-11$ & $50,483,830.83$ & $51,548,580.72$ & 249.91 & 237.72 & $113,648.57$ & $115,571.86$ & 75.65 & 140.5 \\
\hline 8 & $13-19-6-3-13$ & $34,366,841.67$ & $35,091,669.60$ & 603.53 & 574.10 & $381,208.43$ & $387,659.64$ & 171.07 & 289.4 \\
\hline 9 & $3-8-7-5-20$ & $225,675,541.67$ & $230,435,244.00$ & 408.36 & 388.45 & $178,997.11$ & $178,026.29$ & 72.01 & 99.48 \\
\hline 10 & $3-7-5-2-11$ & $24,546,619.17$ & $25,064,329.68$ & 182.75 & 173.84 & $92,206.18$ & $93,766.60$ & 78.95 & 136.6 \\
\hline 11 & $4-10-3-3-9$ & $14,437,912.50$ & $14,242,421.20$ & 125.96 & 119.82 & $53,090.97$ & $53,989.43$ & 38.85 & 67.94 \\
\hline 12 & $6-6-2-4-14$ & $14,330,800.00$ & $14,633,049.60$ & 174.74 & 166.22 & $107,213.98$ & $109,028.37$ & 14.98 & 43.22 \\
\hline 13 & $7-14-5-4-6$ & $7,325,701.26$ & $7,480,206.96$ & 165.03 & 156.99 & $120,672.90$ & $122,715.06$ & 23.51 & 62.54 \\
\hline 14 & $3-8-5-2-18$ & $65,541,501.67$ & $66,923,831.52$ & 228.40 & 217.26 & $77,441.55$ & $78,752.10$ & 7.86 & 21.03 \\
\hline 15 & $4-16-2-5-3$ & $2,756,170.38$ & $2,814,300.51$ & 28.31 & 31.69 & $63,650.47$ & $64,727.63$ & 46.94 & 69.94 \\
\hline 16 & $9-22-8-5-7$ & $33,149,966.67$ & $33,849,129.60$ & 381.40 & 362.80 & $206,028.09$ & $209,514.72$ & 318.29 & 430.6 \\
\hline 17 & $6-6-3-5-19$ & $32,028,186.67$ & $32,703,690.24$ & 296.46 & 282.01 & $189,110.39$ & $187,310.72$ & 11.98 & 22.47 \\
\hline 18 & $8-11-11-4-13$ & $56,616,495.83$ & $57,810,589.20$ & 359.41 & 341.89 & $215,710.08$ & $219,360.56$ & 32.38 & 62.39 \\
\hline 19 & $2-14-6-8-4$ & $679,161.14$ & $653,485.27$ & 15.30 & 14.55 & $7,176.51$ & $7,297.95$ & 1.49 & 6.43 \\
\hline 20 & $8-12-7-4-9$ & $17,331,224.17$ & $17,696,755.44$ & 168.11 & 159.91 & $111,154.18$ & $113,035.25$ & 13.19 & 27.48 \\
\hline 21 & $8-17-7-9-4$ & $7,937,379.12$ & $8,104,785.66$ & 165.48 & 157.42 & $117,867.78$ & $119,862.46$ & 64.70 & 89.53 \\
\hline 22 & $4-9-4-5-9$ & $11,249,867.50$ & $11,180,810.16$ & 150.38 & 143.05 & $52,748.66$ & $53,641.33$ & 19.53 & 31.11 \\
\hline 23 & $3-11-6-6-5$ & $16,189,570.83$ & $16,531,023.60$ & 173.29 & 164.84 & $61,839.49$ & $62,886.00$ & 69.00 & 102.6 \\
\hline 24 & $5-15-4-5-4$ & $5,124,232.07$ & $5,232,306.79$ & 122.26 & 116.30 & $56,065.94$ & $54,980.91$ & 59.43 & 97.94 \\
\hline 25 & $7-7-5-2-14$ & $29,334,570.83$ & $29,953,263.60$ & 331.04 & 314.90 & $171,688.84$ & $174,594.34$ & 25.85 & 73.24 \\
\hline 26 & $3-3-4-5-12$ & $16,754,127.50$ & $17,107,487.28$ & 122.90 & 116.91 & $60,706.26$ & $61,733.60$ & 6.16 & 15.79 \\
\hline 27 & $5-12-7-3-10$ & $36,990,415.00$ & $37,770,576.48$ & 214.42 & 203.96 & $61,023.33$ & $62,056.03$ & 204.21 & 273.6 \\
\hline 28 & $6-12-6-3-7$ & $10,364,970.00$ & $10,583,576.64$ & 182.95 & 174.03 & $92,189.28$ & $91,715.56$ & 33.34 & 51.42 \\
\hline 29 & $3-8-13-4-14$ & $186,635,166.67$ & $190,571,472.00$ & 288.03 & 273.99 & $87,946.30$ & $89,434.62$ & 48.65 & 93.28 \\
\hline 30 & $4-14-7-5-9$ & $68,405,663.33$ & $69,848,400.96$ & 267.78 & 254.72 & $74,752.92$ & $76,017.97$ & 72.99 & 119.2 \\
\hline
\end{tabular}




\section{References}

[1] Srivastava, S.K. (2007), Green supply-chain management: A state-of-the-art literature review, International Journal of Management Reviews, 9(1), 53-80.

[2] Sheu, J.B., Chou, Y.H., and Hu, C.C. (2005), An integrated logistics operational model for green-supply chain management, Transportation Research Part E: Logistics and Transportation Review, 41(4), 287-313.

[3] Sundarakani, B., De Souza, R., Mark, G., Wagner, S.M., and Manikandan, S. (2010), Modeling carbon footprints across the supply chain, International Journal of Production Economics, 128(1), 43-50.

[4] Wang, F., Xiaofan, L., and Shi, N. (2011), A multi-objective optimization for green supply chain network design, Decision Support Systems, 51(2), 262-269.

[5] Mirzapour Ale-e-hashem, S.M.J., Sazvar, Z., Baboli, A., and Rekik, Y. (2012), A MultiObjective Stochastic Model for a Green Supply Chain planning, IFAC Proceedings Volumes, 45(6), 514-521.

[6] Chan, C.K., Lee, Y.C.E., and Campbell, J.F. (2013), Environmental performanceImpacts of vendor-buyer coordination, International Journal of Production Economics, 145(2), 683-695.

[7] Sazvar, Z., Mirzapour Al-e-hashem, S.M.J., Baboli, A., and Akbari Jokar, M.R. (2014), A bi-objective stochastic programming model for a centralized green supply chain with deteriorating products, International Journal of Production Economics, 150, 140-154.

[8] Fahimnia, B., Sarkis, J., Dehghanian, F., Banihashemi, N., and Rahman, Sh. (2014), The impact of carbon pricing on a closed-loop supply chain: an Australian case study, Journal of Cleaner Production, 59, 210-225.

[9] Baud-Lavigne, B., Agard, B., and Penz, B. (2014), Environmental constraints in joint product and supply chain design optimization, Computers \& Industrial Engineering, 76, $16-22$.

[10] Azadeh, A., Raoofi, Z., and Zarrin, M. (2015), A multi-objective fuzzy linear programming model for optimization of natural gas supply chain through a greenhouse gas reduction approach, Journal of Natural Gas Science and Engineering, 26, 702-710.

[11] Li, B., Zhu, M., Jiang, Y., and Li, Z. (2016), Pricing policies of a competitive dualchannel green supply chain, Journal of Cleaner Production, 112(3), 2029-2042.

[12] Kannan, D., Khodaverdi, R., Olfat, L., Jafarian, A., and Diabat, A. (2013), Integrated fuzzy multi criteria decision making method and multi-objective programming approach for supplier selection and order allocation in a green supply chain, Journal of Cleaner Production, 47, 355-367.

[13] Kadziński, M., Tervonen, T., Tomczyk, M.K., and Dekker, R. (2017), Evaluation of multi-objective optimization approaches for solving green supply chain design problems, Omega, 68, 168-184.

[14] Niakan, F., Baboli, A., Botta-Genoulaz, V., Tavakkoli-Moghaddam and R., Camapgne, J.P. (2013), A Multi-Objective Mathematical Model for Green Supply Chain Reorganization, IFAC Proceedings Volumes, 46(7), 81-86.

[15] Kostin, A., Guillén-Gosálbez, G., and Jiménez, L. (2015), Dimensionality reduction applied to the simultaneous optimization of the economic and life cycle environmental performance of supply chains, International Journal of Production Economics, 159, 223232.

[16] Xing, K., Qian, W., and Zaman, A.U. (2016), Development of a cloud-based platform for footprint assessment in green supply chain management, Journal of Cleaner Production, 139, 191-203. 
[17] Yu, F., Xue, L., Sun, Ch., and Zhang, Ch. (2016), Product transportation distance based supplier selection in sustainable supply chain network, Journal of Cleaner Production, 137, 29-39.

[18] Shaw, K., Irfan, M., Shankar, R., and Yadav, S.S. (2016), Low carbon chance constrained supply chain network design problem: a Benders decomposition based approach, Computers \& Industrial Engineering, 98, 483-497.

[19] Haimes Y.Y, Lasdon, L.S., and Wismer D.A. (1971), On a bi-criterion formulation of the problems of integrated system identification and system optimization, IEEE Transactions on Systems, Man, and Cybernetics 1, 296-297.

[20] Pasandideh, S.H.R., Akhavan niaki, S.T., and Asadi, K. (2015), Optimizing a bi-objective multi-product multi-period three echelon supply chain network with warehouse reliability, Expert Systems with Applications, 42, 2615-2623.

[21] Sharma, D.K., Ghosh, D., and Mattison, D.M. (2003), An application of goal programming with penalty functions to transhipment problems, International Journal of Logistics: Research and Applications, 6, 125-136.

[22] Che, Z.H., and Chiang, C.J. (2010), A modified Pareto genetic algorithm for multiobjective build-to-order supply chain planning with product assembly, Advances in Engineering Software, 41(7-8), 1011-1022.

[23] Nurjanni, K.P., Carvalho, M.S., and Costa, L. (2017), Green supply chain design: a mathematical modelling approach based on a multi-objective optimization model, International Journal of Production Economics, 183(B), 421-432.

[24] Kaliszewski, I. (1987), A modified weighted Tchebycheff metric for multiple objective programming, Cmpur. Opris Rus, 14(4), 315-323. 